\title{
Artroplastia minimamente invasiva do joelho
}

\author{
Minimally invasive total knee arthroplasty
}

\author{
Geraldo Rocha Motta Filho ${ }^{1}$, NaAson Cavanellas ${ }^{2}$
}

\section{RESUMO}

A artroplastia total do joelho é, tradicionalmente, realizada através de um acesso anterior de aproximadamente $20 \mathrm{~cm}$ de comprimento, utilizando uma artrotomia no intervalo entre os músculos reto femoral e vasto medial. Esse acesso fornece excelente exposição articular, mas interfere com a integridade suprapatelar, o que poderia ser responsável por aderências que dificultariam a recuperação mais precoce da flexão do joelho. Diversas publicações concluíram que os cirurgiões estão mais satisfeitos que seus pacientes com os resultados clínico-funcionais das artroplastias totais do joelho. Dessa forma, a utilização de um acesso minimamente invasivo poderá proporcionar a redução do período de hospitalização, diminuir a intensidade da dor pós-operatória, reduzindo o tempo de recuperação da amplitude de movimentos e o retorno da função. Foram discutidas as diversas técnicas propostas para a realização de uma artroplastia com técnica minimamente invasiva e concluiu-se que o desenvolvimento dessa técnica pode ser difícil e consumir tempo, mas os benefícios e satisfação dos pacientes irão suplantar esse esforço extra.

1. Mestre, Chefe de Clínica da Divisão de Ortopedia e Traumatologia e Chefe do Serviço de Cirurgia do Ombro e Cotovelo do Instituto Nacional de Traumatologia e Ortopedia - INTO - Rio de Janeiro (RJ), Brasil.

2. Chefe Substituto do Serviço de Cirurgia do Joelho do Instituto Nacional de Traumatologia e Ortopedia - INTO - Rio de Janeiro (RJ), Brasil.

Endereço para correspondência: Geraldo Rocha Motta Filho, Rua Ferreira de Resende, 14, apto. 302, Lagoa - 22471-170 - Rio de Janeiro, RJ. E-mail: geraldomotta@terra.com.br

Copyright RBO2007
Essas mudanças necessitam de estudos clínicos que possam documentar a sua eficácia, porém, desde já, os números existentes, apesar de pequenos, já demonstram os benefícios claros do acesso subvasto minimamente invasivo.

Descritores - Joelho; Artroplastia do joelho; Artroplastia do joelho/ instrumentação; Procedimentos cirúrgicos minimamente invasivos; Recuperação de função fisiológica

\section{ABSTRACT}

Total knee arthroplasty is traditionally performed through an anterior access of approximately $20 \mathrm{~cm}$, arthrotomy being made at the interval between the rectus femoris and the midvastus muscles. The access provides excellent joint exposure, but it does interfere with suprapatellar integrity, which could lead to adherences that impair earlier of knee flexion recovery. Several publications have concluded that surgeons are more pleased than their patients with the clinical and functional results of total knee arthroplasties. The use of a minimally invasive access may provide a shorter hospitalization period, decreased post-operative pain intensity thus reducing the time required for movement amplitude recovery and the return of function. The different techniques proposed to perform minimally invasive arthroplasties have been discussed, and conclusion is that the development of such a technique may be difficult and take time, but the benefits and patient satisfaction will offset such added effort. The changes require clinical studies to document their effectiveness, but the results already obtained, though with small series, show that there are clear benefits to be achieved with a minimally invasive subvastus access.

\footnotetext{
Keywords - Knee; Arthroplasty, replacement, knee; Arthroplasty, replacement, knee/instrumentation; Surgical procedures, minimally invasive; Recovery of function
} 


\section{INTRODUÇÃO}

A primeira artroplastia total do joelho (ATJ) foi realizada em 1974(1-2). Desde então, está em permanente evolução, mudando e melhorando, apesar de ser um dos dois procedimentos ortopédicos mais bem-sucedidos na história da especialidade ao lado da artroplastia do quadril ${ }^{(3-5)}$. Os cirurgiões, bioengenheiros e especialistas nos instrumentais e implantes utilizados continuam pesquisando para que esse procedimento possa estar à disposição de pacientes mais jovens, ativos e com objetivos especiais.
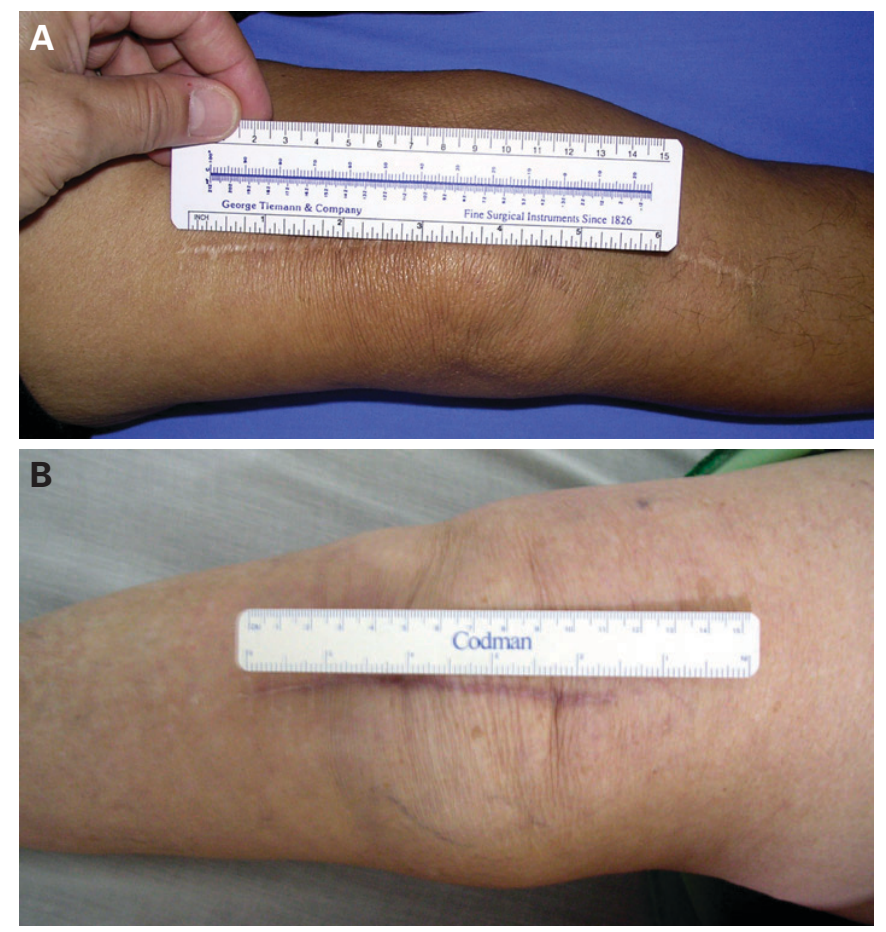

Figura 1 - A e B) Acesso convencional x "limitado"

A técnica convencional para a realização de uma artroplastia total do joelho inicia-se com uma incisão na pele de aproximadamente 20 a $25 \mathrm{~cm}$, artrotomia medial, eversão da patela, distúrbio do mecanismo extensor do joelho, luxação da articulação, além de violação extensa das partes moles ao redor da articulação $0^{(3-5)}$.

Laskin $^{(6)}$ e outros, no final dos anos 90, passaram a questionar a recuperação funcional dos pacientes, sabendo-se que seria penosa. Dentre as causas discutidas, podemos destacar: a proximidade do tendão do quadríceps e sua secção poderiam ser responsáveis pelo tempo de recuperação da função muscular; a destruição da região suprapatelar seria responsável pelo surgimento de aderências que dificultariam o ganho da amplitude de movimentos; a manutenção do joelho em posição de hiperflexão prolongada poderia ser responsável pelo surgimento de fibrose nas partes moles contribuindo também para dificultar o ganho da amplitude de movimentos; rotação de $180^{\circ}$ do mecanismo extensor poderia causar microrrupturas no quadríceps e ligamento patelar: e, finalmente, a combinação de todos esses fatores contribuiria para uma recuperação funcional difícil ${ }^{(7)}$.

Avaliações funcionais mais sofisticadas do desempenho da articulação do joelho, realizadas mais recentemente, que envolvem questionamentos com dados subjetivos e objetivos, demonstram que os cirurgiões estão mais satisfeitos que os seus pacientes. Isso sugere que o desenvolvimento de novas técnicas cirúrgicas e de desenho dos implantes e do instrumental necessita de investigação para que possamos atingir os objetivos dos nossos pacientes ${ }^{(8)}$.

As mais variadas especialidades cirúrgicas nos últimos anos desenvolveram técnicas chamadas de cirurgias minimamente invasivas.

A realização da artroplastia unicompartimental do joelho permitiu a diminuição considerável da extensão do acesso cirúrgico, Repicci e o grupo da Oxford University foram os autores da conceituação de cirurgia minimamente invasiva (CMI) do joelho, obtendo resultados comparáveis aos da técnica clássica $^{(9-10)}$.

Infelizmente, até essa data não existia uma definição de CMI com parâmetros claros para o joelho. A extensão da incisão, acessos que não violam o mecanismo extensor do joelho, redução do tempo de hospitalização, diminuição do percentual de transfusão sanguínea e retorno mais precoce da mobilidade e função da articulação são parâmetros que caracterizam o procedimento ${ }^{(7,11-12)}$.

A artroplastia total do joelho, quando realizada com acesso e exposição da articulação limitados, implica maior dificuldade técnica para o cirurgião, se comparada com o acesso cirúrgico convencional. Dessa forma o sucesso da artroplastia realizada com técnica minimamente invasiva exige do cirurgião intimidade e habilidade na utilização do instrumental e dos implantes.

O nosso objetivo é apresentar uma revisão dos acessos e procedimentos popularizados na realização da artroplastia total minimamente invasiva do joelho e discutir os resultados apresentados na literatura médica. 


\section{INDICAÇÕES PARA A CMI}

Existem limitações para utilização da técnica. Para a maioria dos autores, os pacientes devem apresentar deformidades limitadas a $15^{\circ}$ de varo, $20^{\circ}$ de valgo e contratura em flexão de até $10^{\circ(11)}$. O arco de movimento mínimo deverá ser de $90^{\circ}$. Além desses parâmetros, pacientes portadores de artrite reumatóide, diabetes ou que foram submetidos a cirurgias prévias na articulação devem ser excluídos do rol de indicações para a CMI.

A experiência clínica demonstrou que pacientes musculosos, obesos, portadores de fêmures com dimensões avantajadas e patela baixa também dificultam a utilização dessa técnica.

\section{OPÇÕES DE ACESSOS PARA A CMI}

Serão descritas a seguir as opções de acessos cirúrgicos que têm sido utilizadas na prática cirúrgica.

\section{Acesso convencional limitado}

Esse acesso modifica a prática do acesso tradicional, com uma incisão cutânea que varia de 10 a $14 \mathrm{~cm}$, estendendo-se do pólo superior da patela até a tuberosidade anterior da tíbia (TAT). Recomenda-se que o tamanho da incisão seja a menor possível, mas que deverá estender-se o necessário para permitir visualização adequada dos três compartimentos do joelho e não acarretar sofrimento da pele. Após a incisão da pele, os retalhos cutâneos são mobilizados para que se realize o "conceito das janelas" que permitem visualização adequada da articulação(13-15).

O ponto mais importante desse tipo de acesso é a extensão da artrotomia medial. Ela deverá estender-se de 2 a $4 \mathrm{~cm}$ no tendão do quadríceps. Isso, em geral, permite que a patela seja deslocada lateralmente sem realizarmos a sua eversão. Se existir dificuldade para tal, ou a inserção do tendão patelar estiver sob risco, a artrotomia pode ser facilmente prolongada.

A utilização do instrumental clássico para a realização dos cortes ósseos é possível, mas dificulta o procedimento. Atualmente, existem instrumentais específicos disponíveis para as CMI, com dimensões mais reduzidas, o que permite posicionamento mais fácil e técnica mais precisa.

A seleção do tipo, tamanho e rotação dos implantes, os cortes ósseos e a liberação das partes moles são realizados observando-se os mesmos parâmetros claramente estabelecidos na literatura médica, cuja discussão não é o objetivo deste artigo.
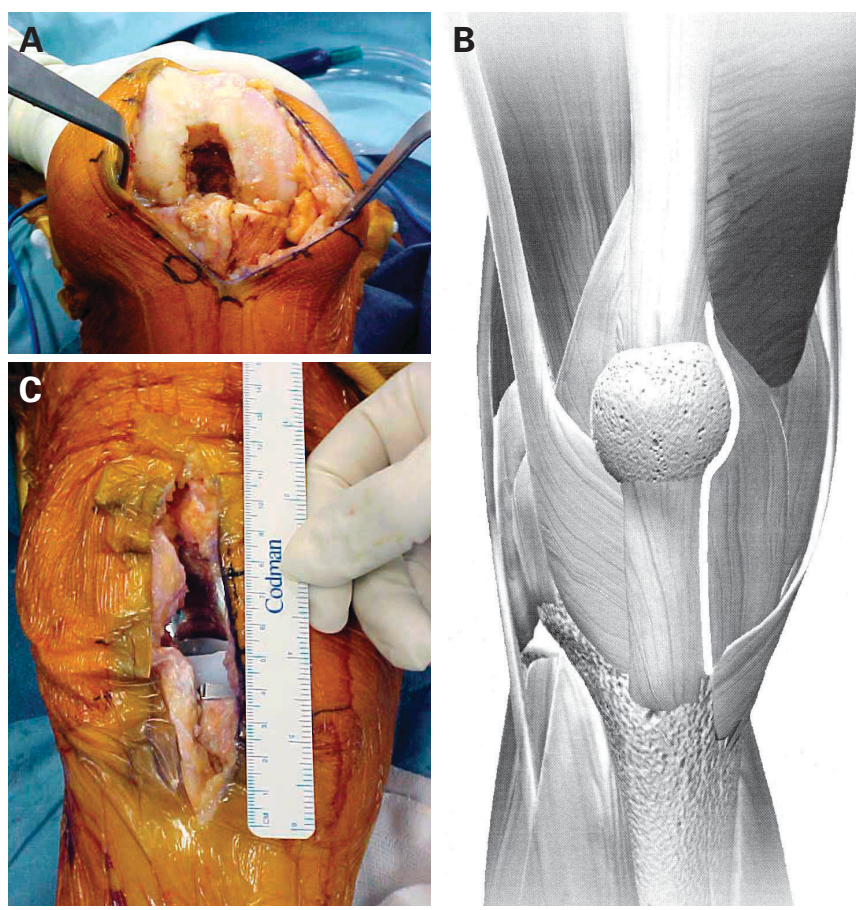

Figura 2 - A, B e C) Acesso convencional limitado popularizado por Scuderi

\section{Minimidvasto}

A técnica consiste numa variação do acesso clássico midvasto descrito por Engh et al $^{(16-17)}$. A incisão cutânea é realizada um pouco mais medial do que o convencional, prolongando-se do pólo proximal da patela até a TAT.

Após a dissecção dos retalhos cutâneos, o joelho é flexionado em torno de $70^{\circ}$, o que permitirá a visualização do músculo vasto medial. Uma artrotomia medial é realizada estendendo-se de forma romba ao músculo vasto medial, que será dividido ao longo de suas fibras em uma extensão de 1,5 a $2,0 \mathrm{~cm}$. Em seguida, um afastador curvo de Hohmann é colocado embaixo da patela com o joelho em extensão. Há liberação da cápsula inferior na região ântero-lateral da tíbia e ressecção parcial da gordura de Hoffa associada à liberação capsular sob o mecanismo extensor, permitindo o deslocamento lateral da patela por diante do côndilo femoral lateral. O joelho então será flexionado entre 60 e $90^{\circ}$, o que permitirá a exposição da extremidade distal do fêmur e a realização do procedimento cirúrgico ${ }^{(6-7,12,18)}$.

A cimentação dos componentes nas técnicas com acesso limitado é sempre um desafio. Deveremos começar pela tíbia, fêmur e, por último, o componente patelar. Cabe ressaltar que componentes tibiais com uma haste mais longa poderão dificultar a colocação do implante. 


\section{"Quad-sparing approach"}

Uma vez mais, reconhecendo-se a importância do mecanismo extensor para a reabilitação e função do joelho, uma técnica cirúrgica foi desenvolvida para a sua preservação.

$\mathrm{O}$ acesso chamado de quad-sparing approach consiste de uma artrotomia medial que não se estende ao tendão do quadríceps, fibras do músculo vasto medial ou intervalo subvasto. Em virtude da limitação da artrotomia, a patela não poderá ser deslocada lateralmente por diante do côndilo femoral lateral. Isso significa que os tempos clássicos do procedimento cirúrgico que são realizados em hiperflexão sejam feitos mais em extensão para relaxar o mecanismo extensor e oferecer acesso à extremidade distal e anterior do fêmur e proximal da tíbia. A realização dos cortes ósseos necessita de instrumental específico e apresenta fundamentos diversos da prática convencional da artroplastia total do joelho ${ }^{(19-20)}$.
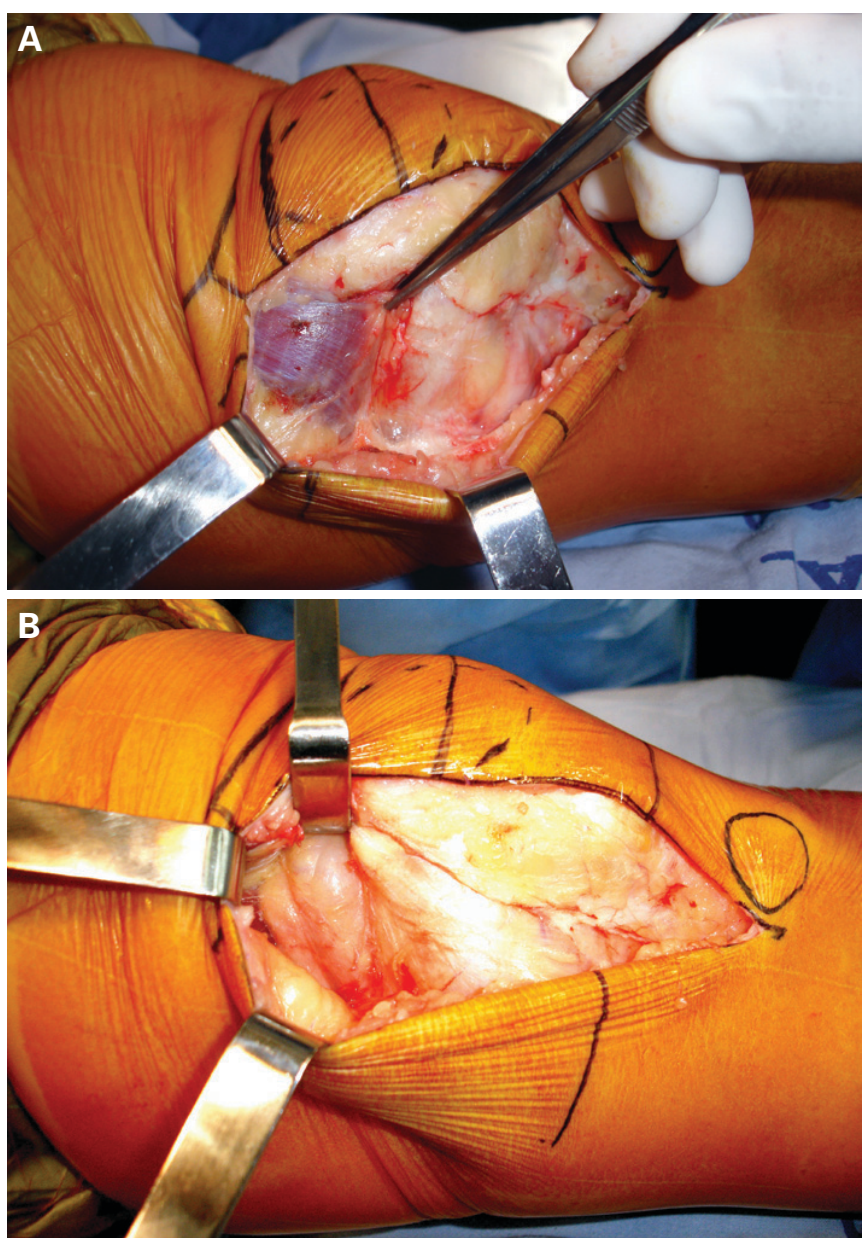

Figura 3 - A e B) Acesso minissubvasto
$\mathrm{O}$ acesso foi realizado inicialmente em pacientes selecionados, com bom arco de movimento do joelho, baixo índice de massa corporal e que permaneceram em média pouco mais de dois dias internados.

\section{Minissubvasto}

A prática do acesso subvasto tradicional nunca se tornou uma técnica popular em razão da dificuldade em everter a patela nos indivíduos brevilíneos, obesos e musculosos, que representam número significativo dos pacientes que precisam ser submetidos à artroplastia total do joelho, isso apesar de a literatura demonstrar ser procedimento menos doloroso e que apresenta melhor função do quadríceps no pós-operatório imediato $^{(21-22)}$
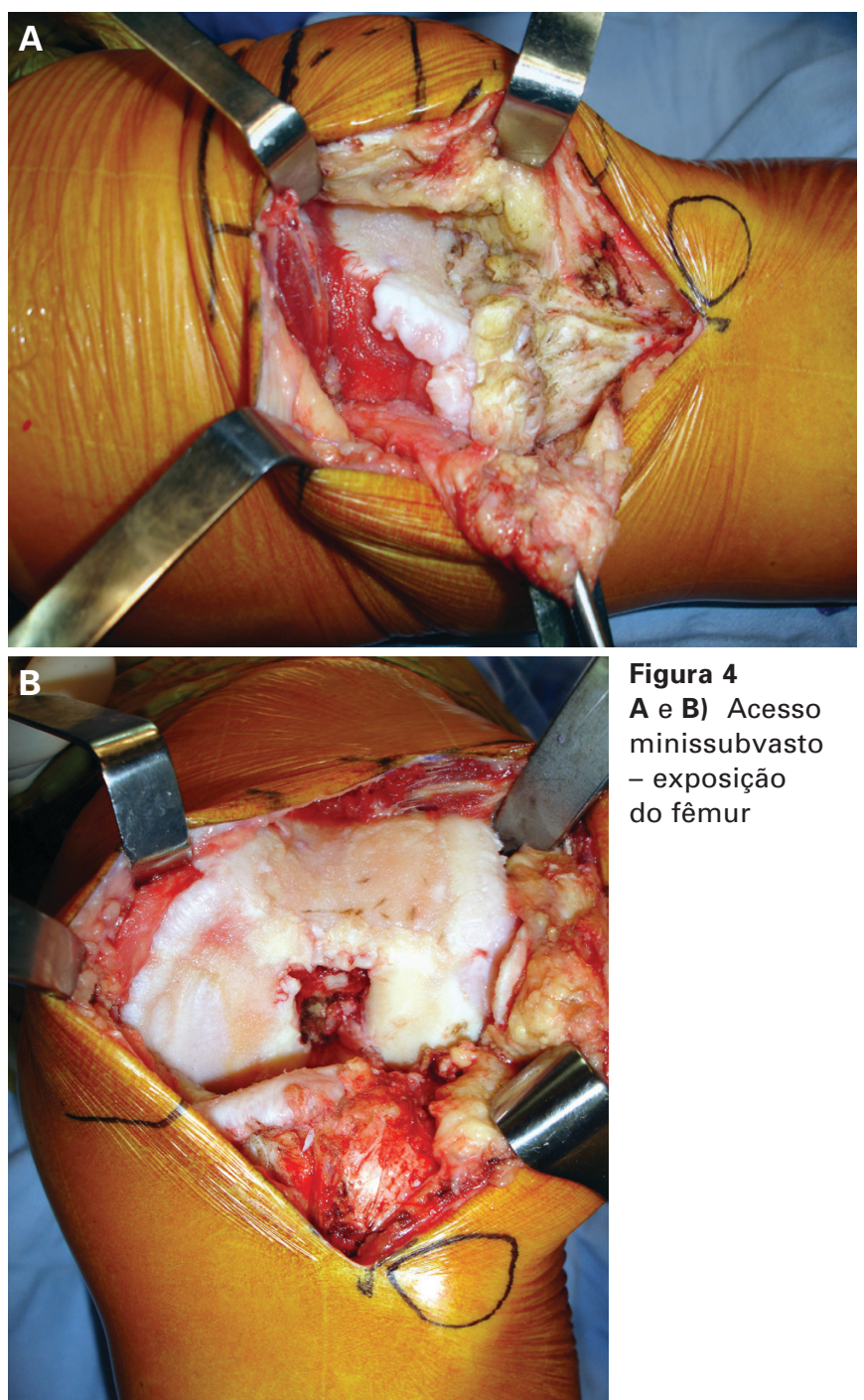

Figura 4

A e B) Acesso minissubvasto - exposição do fêmur 
Por outro lado, a modificação do acesso subvasto popularizado por Pagnano et $a l^{(11)}$ permite, com incisão cutânea limitada, ótima exposição da articulação, sem eversão da patela, mínimo distúrbio da região suprapatelar e do mecanismo extensor. A patela será deslocada lateralmente, possibilitando a visualização dos côndilos femorais e planalto tibial, o que permite a realização dos cortes ósseos e o balanço de partes moles de forma segura.

Um afastador de Hohmann, estreito, com curvatura de $90^{\circ}$ é colocado na goteira lateral. O joelho é flexionado a praticamente $90^{\circ}$, o que proporciona ótima visualização da extremidade distal do fêmur e permite que os cortes ósseos sejam realizados.
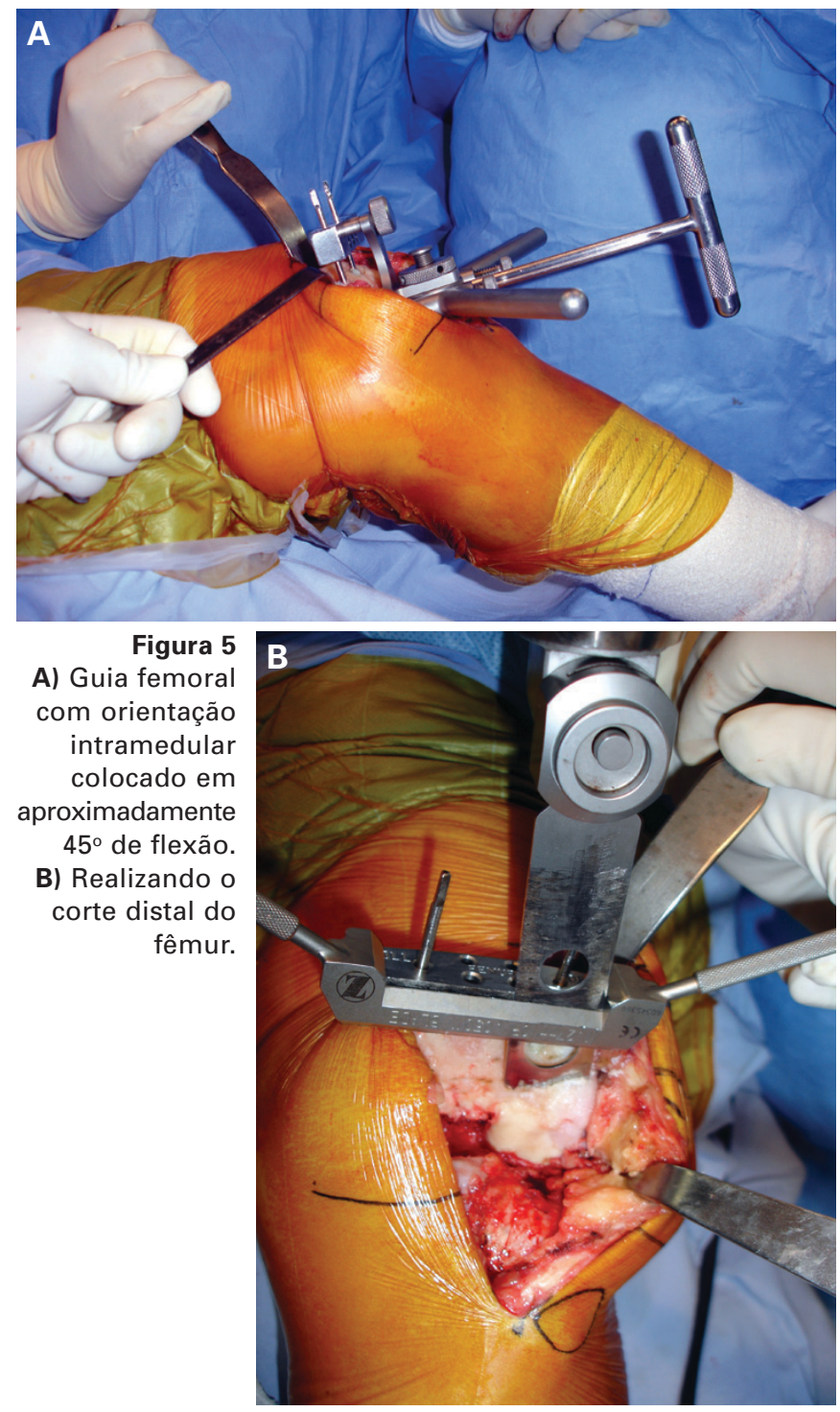

O corte tibial é realizado utilizando-se um guia extramedular e lâmina da serra estreita, porém espessa, o que possibilita corte mais preciso. A lâmina mais estreita oferece maior maleabilidade para se trabalhar num acesso mais limitado.

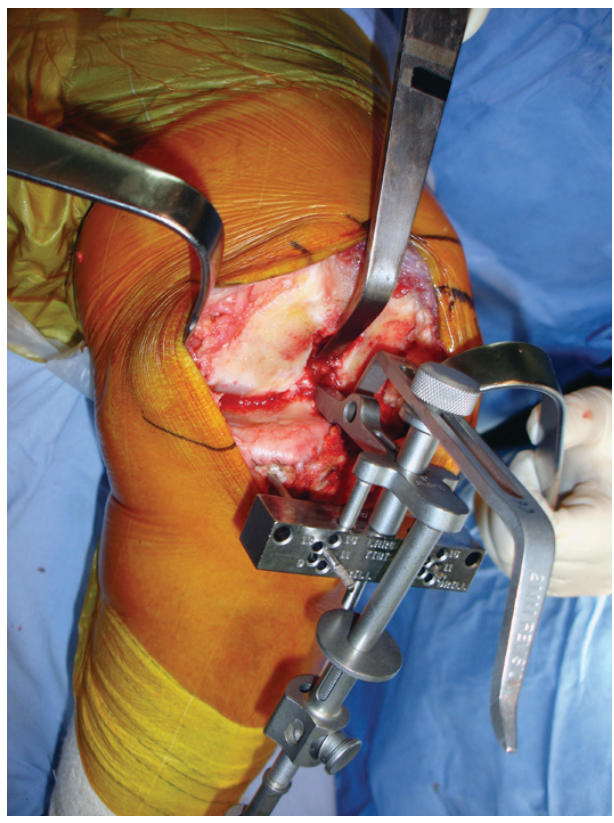

\section{Figura 6}

Na tíbia

utiliza-se o guia respeitando-se os parâmetros clássicos

Em seguida, com o joelho em $60^{\circ}$ de flexão, é possível determinar o tamanho e rotação do componente femoral, pois nessa posição pode ser feita uma avaliação precisa da cortical anterior do fêmur e a seleção do tamanho do implante, tendose a certeza de que a cortical anterior do fêmur não será violada. A rotação poderá ser conferida utilizando-se os parâmetros convencionais, tais como o eixo transepicondilar e a linha de Whitesides.

A patela poderá, com esse acesso, ser preparada ao final do procedimento. Não é necessário o corte da patela no início do procedimento, só para aumentar o acesso cirúrgico, evitando assim o eventual comprometimento de sua superfície durante o ato cirúrgico. Ela poderá ser preparada com a técnica da "mão livre" ou utilizando-se um guia, dependendo da preferência do cirurgião, não sendo necessária sua eversão.

O primeiro componente a ser cimentado será a bandeja tibial metálica, em seguida, o fêmur e, finalmente, a patela. Deveremos ter o cuidado de retirar todo o excesso de cimento, mesmo que seja um pouco mais difícil a sua realização. Nesse momento, o polietileno tibial definitivo pode ser colocado $^{(11)}$. 


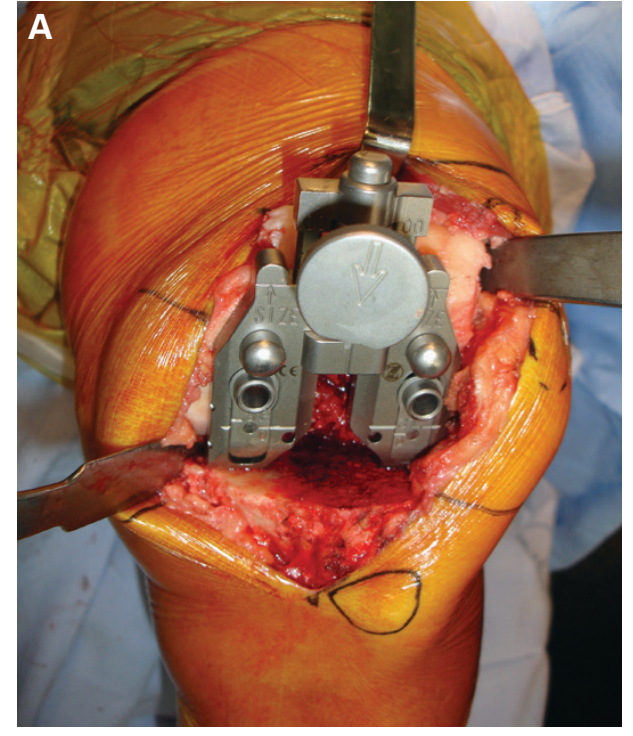

A) Estabelecendo a rotação do componente femoral.

B) Determinando o tamanho e o posicionamento do componente

femoral.
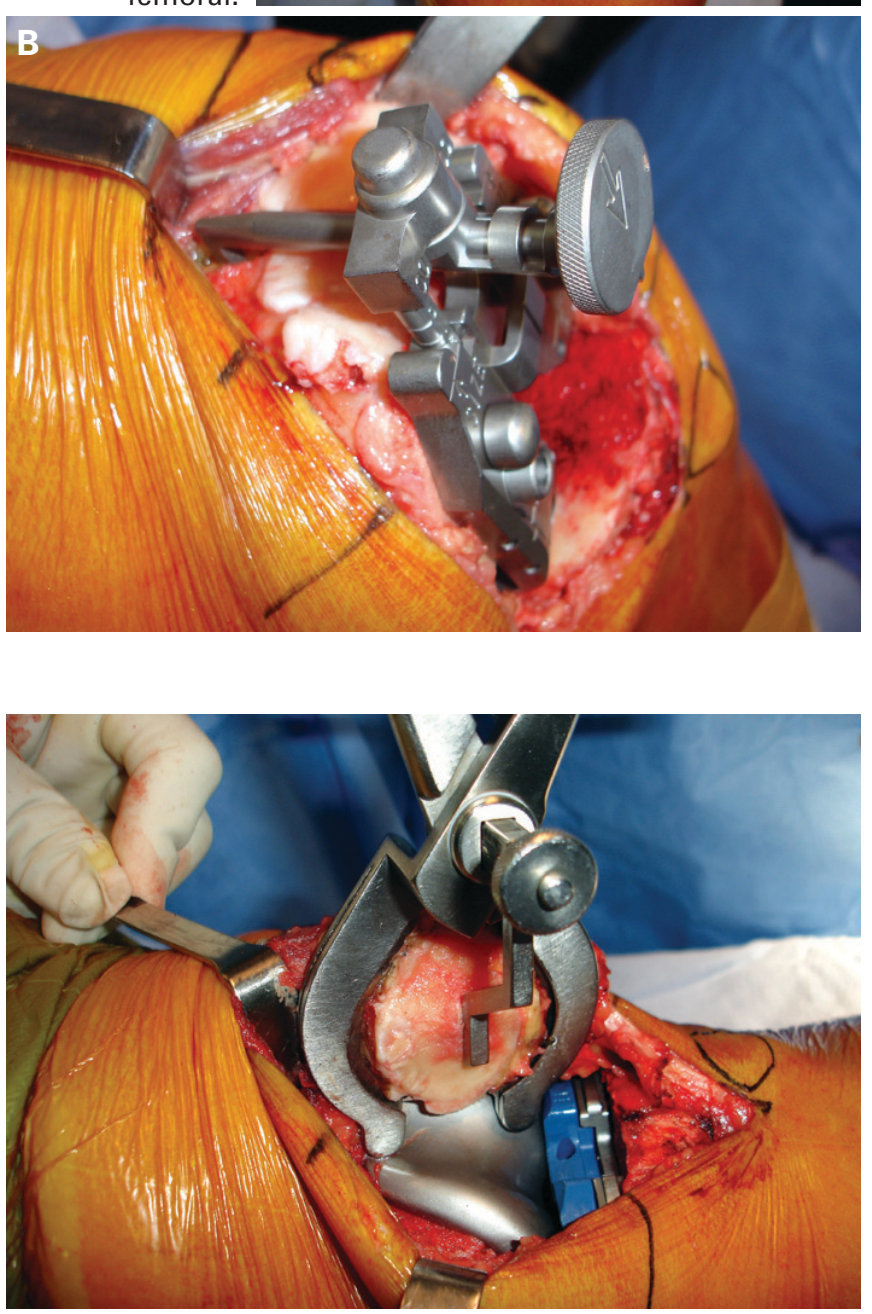

Figura 8 - Preparo da patela sem que seja realizada a sua eversão
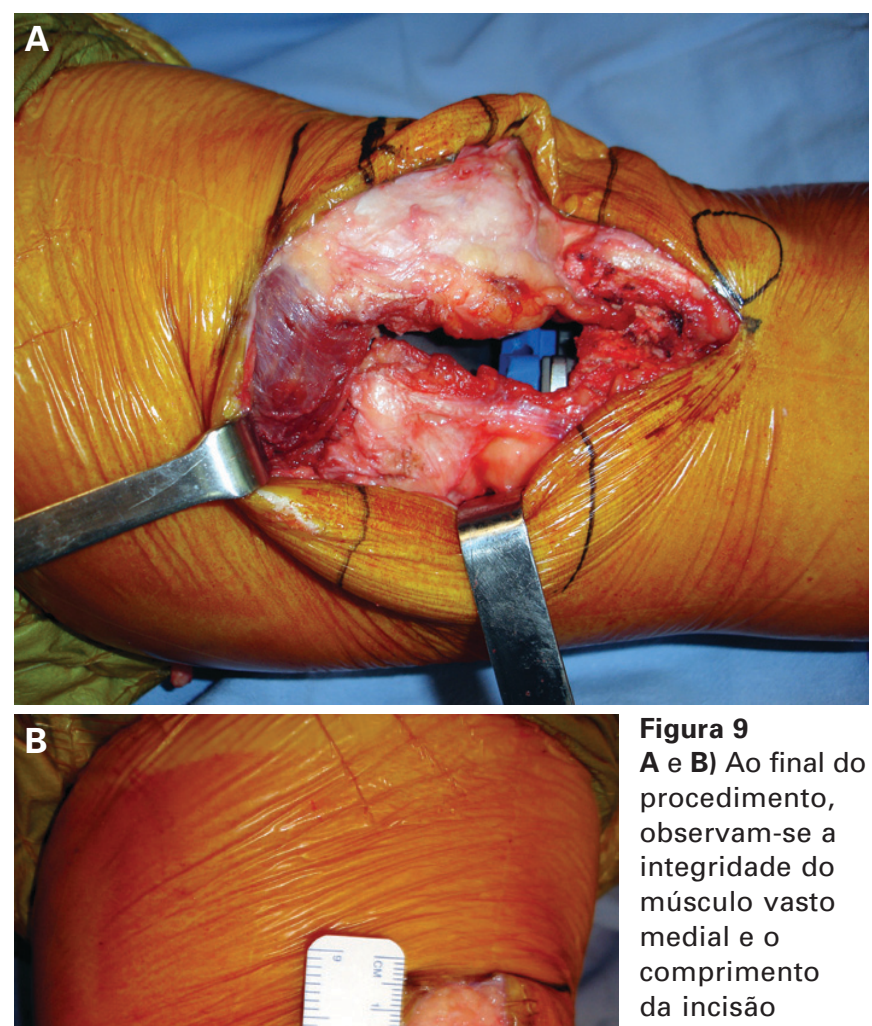

\section{DISCUSSÃO}

Claramente, a artroplastia total do joelho com acesso limitado é um procedimento que exige técnica mais apurada do que a praticada com o acesso convencional. Inicialmente, exige tempo cirúrgico mais prolongado e risco maior da ocorrência de complicações.

Noble et $\mathrm{al}^{\left({ }^{(8)}\right.}$ salientaram a preocupação com os resultados funcionais das artroplastias totais do joelho. Esses autores determinaram o fator idade na função do joelho com a aplicação de questionários sofisticados que mensuraram a função 
articular. Foram avaliados também pacientes submetidos à artroplastia. Chegaram à conclusão de que existem diferenças substanciais na capacidade funcional entre os grupos submetidos à artroplastia e aqueles só comprometidos pela idade. Em torno de 52\% dos pacientes submetidos à artroplastia relataram algum grau de limitação na realização das atividades funcionais, ao contrário de somente $22 \%$ dos não operados. Além do mais, concluíram que somente $40 \%$ do déficit funcional relatado pelos pacientes submetidos à artroplastia total pode ser atribuído ao comprometimento fisiológico normal determinado pela idade. Isso nos permite concluir que o desenvolvimento de técnicas como as propostas por aqueles que defendem a CMI têm de merecer a atenção de centros especializados em artroplastia, para que no futuro possam ser transferidos a experiência e os parâmetros que devem ser aplicados na prática clínica aos cirurgiões generalistas responsáveis por grande número das artroplastias.

Mas, infelizmente, a divulgação da CMI ultrapassou os parâmetros e limites recomendáveis para o desenho, avaliação e liberação de uma nova técnica e pode ter sido utilizada indiscriminadamente na prática médica. Mas esse fato não pode constituir barreira para a utilização de uma técnica e, sim, ser reconhecido pela comunidade científica, considerando-se a importância e a necessidade da evolução da ciência ${ }^{(23)}$.

$\mathrm{O}$ acesso convencional limitado foi desenvolvido para diminuir a morbidade na artroplastia total do joelho e melhorar o pós-operatório imediato, tempo de hospitalização, percentual de transfusão sanguínea e a reabilitação ${ }^{(14-15)}$. Essa técnica representa uma transição para a CMI, mas, por utilizar os mesmos parâmetros anatômicos e técnica do procedimento convencional, permitirá que maior número de cirurgiões possa utilizá-la com mais segurança, uma vez que não se propõe que eles mudem radicalmente o que vinha sendo sua prática anterior.

Haas et $a l^{(18)}$ relataram sua experiência em 335 pacientes submetidos à artroplastia total do joelho, em que o procedimento cirúrgico foi realizado pelo acesso do tipo minimidvasto. Foi constatada a predominância de mulheres sobre os homens, com um arco de movimento médio no pré-operatório de $109^{\circ}$. O arco de movimento após seis semanas foi de $111^{\circ}, 121^{\circ}$ e $125^{\circ}$ após um ano. O percentual de complicações com essa técnica foi igual ao que ocorre quando o acesso é realizado de forma convencional.

Laskin $^{(24)}$ relatou sua experiência com o acesso minimidvasto em 34 pacientes, comparando-os com 26 operados com acesso parapatelar medial convencional. A incisão cutânea média no grupo da CMI foi de $12,8 \mathrm{~cm}$. Ao final da sexta sema- na de pós-operatório, a mudança no escore para avaliação do joelho do Hospital for Special Surgery apresentava valores superiores e estatisticamente significativos no grupo submetido à CMI, assim como os valores médios na escala analógica da dor e a quantidade de medicação analgésica, que também foram menores. O alinhamento radiográfico e o posicionamento dos implantes não apresentaram alterações em ambos os grupos.

A preocupação que existe com o acesso midvasto em relação à possível lesão neurológica do músculo vasto medial não se confirmou na prática clínica. Kelly et al ${ }^{(25)}$ demonstraram, em estudo que comparou o acesso convencional com o midvasto, que as alterações eletromiográficas que ocorrem no quadríceps com esse acesso não revelam consequiências funcionais e representam neuropraxia reversível, que até pode ser evitada por uma dissecção romba e limitada a não mais que $2 \mathrm{~cm}$, como recomendado pelos autores que apresentam experiência com essa técnica ${ }^{(6,26-27)}$.

Tria $e a^{(28)}$ e Alan et al ${ }^{(29)}$ relataram seus resultados utilizando o acesso tipo quad-sparing, que foram comparáveis aos dos demais autores que empregaram os outros acessos na CMI. Em 70 pacientes com idade média de 67 anos, o tempo de internação foi de quatro dias e, nos últimos doentes operados, de até dois dias. As radiografias pós-operatórias mostraram valgo femoral médio de $4^{\circ}$ e varo tibial de $2,5^{\circ}$, com alinhamento médio de $4^{\circ}$. Esse grupo foi comparado com outro operado no mesmo período, utilizando-se a técnica convencional, não existindo nenhuma diferença nos parâmetros avaliados que apresentasse significância estatística. $\mathrm{O}$ arco de movimento na primeira visita pós-operatória foi $20^{\circ}$ maior quando comparado com o desse mesmo grupo mencionado anteriormente.

Apesar dos resultados descritos, esse acesso nos dias de hoje é utilizado por poucos grupos em virtude da dificuldade técnica na sua execução, necessidade de instrumental específico, os cortes ósseos sendo realizados praticamente em extensão e de medial para lateral, o que caracteriza conceitos diversos estabelecidos para a realização de uma ATJ. Dessa forma, a técnica ficou restrita a um pequeno número de cirurgiões.

Pagnano et $a l^{(30)}$ realizaram estudo anatômico da inserção do músculo vasto medial na patela e verificaram que, na imensa maioria dos joelhos estudados, a inserção ocorre no terço médio da patela inclinada a $45^{\circ}$. Dessa forma, qualquer tipo de artrotomia irá seccionar um segmento do tendão do vasto medial. Pode-se então afirmar que qualquer tipo de acesso recomendado na literatura não pode ser dito quad-sparing, como proposto por alguns autores. 


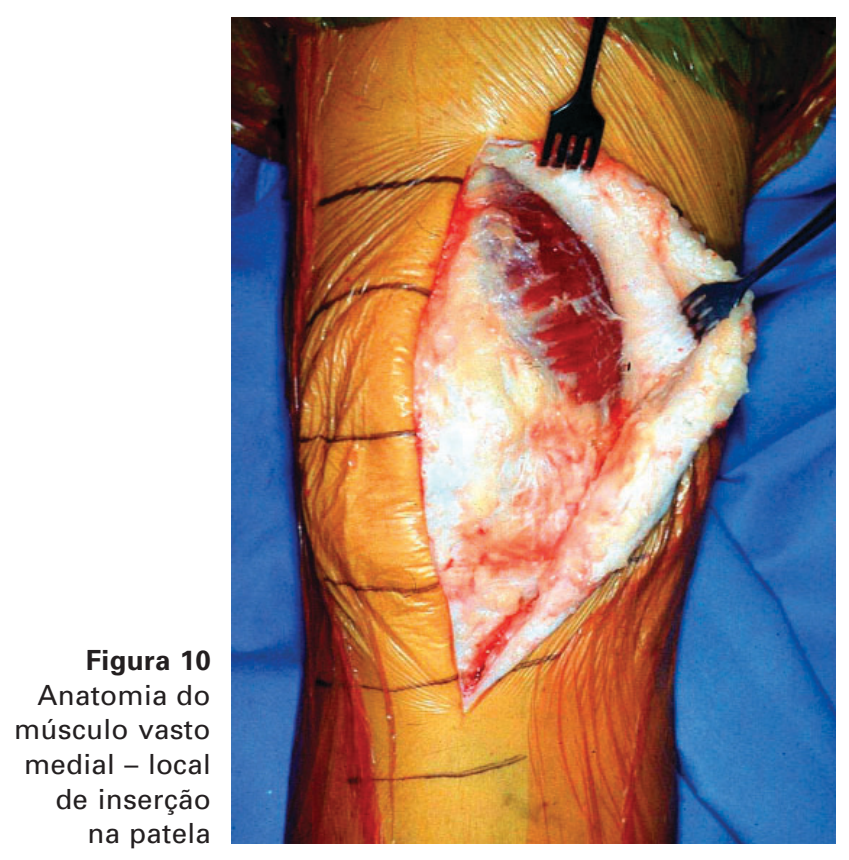

Pagnano et al $^{(11)}$ apresentaram a experiência da Clínica Mayo em 103 pacientes consecutivos que apresentavam artrose primária do joelho e que foram submetidos à artroplastia total do joelho utilizando-se o acesso subvasto modificado pelos autores. A idade média do grupo era de 66 anos, predominância do sexo feminino, tempo cirúrgico médio de 58 minutos e tempo médio de hospitalização de 2,8 dias. Todos os pacientes foram operados sem que existisse necessidade de conversão do acesso utilizando-se um implante que sacrifica o ligamento cruzado posterior. Todos os pacientes foram avaliados minuciosamente em relação ao posicionamento dos componentes femoral e tibial. Todos os componentes femorais foram posicionados a $6^{\circ} \pm 2^{\circ}$ de valgo, com exceção de dois pacientes, cujos fêmures se encontravam a $9^{\circ}$ de valgo. Os implantes tibiais foram posicionados a $90^{\circ} \mathrm{em}$ relação ao eixo longitudinal da tíbia, com exceção de um paciente com $3^{\circ}$ de valgo. As complicações locais, de cicatrização da ferida, ocorreram em dois pacientes, sem comprometimento do resultado final. $\mathrm{O}$ arco de movimento foi de $116^{\circ}$ com oito semanas e de $119^{\circ}$ ao final de um ano.

Aglietti et $\mathrm{l}^{(31)}$ realizaram a comparação do acesso tipo quadríceps sparing com o minissubvasto. Concluíram que os dois acessos não apresentaram diferenças com relação aos parâmetros do pós-operatório imediato e de resultados funcionais precoces. Dessa forma, não existiria razão para a realização do acesso tipo quadriceps sparing em vez do minissubvasto, já que o primeiro é de muito mais difícil realização técnica e, portanto, podendo estar associado a maior número de complicações relacionadas ao método.

Lonner ${ }^{(32)}$ avaliou os resultados publicados que utilizaram vários tipos de acesso para a CMI. Concluiu que até hoje os trabalhos não apresentam representatividade estatística que permita afirmar qual seria o acesso mais recomendado. De toda forma, concluiu que os dados apresentados permitem afirmar que a técnica minimamente invasiva é superior ao acesso convencional, devendo-se no futuro avaliar por meio de ferramentas sofisticadas e, até mesmo, deduzir que existem diferenças entre os benefícios a curto e longo prazo entre a CMI e a cirurgia convencional.

\section{CONCLUSÃO}

O acesso minissubvasto é confiável e reprodutível para a realização de uma artroplastia total do joelho. É um método eficiente quando o procedimento se realiza por um acesso limitado. A técnica poderá ser utilizada por um cirurgião habilitado na técnica convencional, que poderá progressivamente diminuir seu acesso. Finalmente, o acesso minissubvasto parecenos lógico do ponto de vista anatômico e, nos dias de hoje, já existem números que mostram claro benefício da técnica minimamente invasiva, mesmo que ainda com séries que representam pequeno número de pacientes e com tempo de seguimento não comparável com as séries clássicas que estabeleceram a sobrevida de uma artroplastia total do joelho(1-2,11).

\section{CRÍTICA}

A idéia dos procedimentos minimamente invasivos é envolvente e desejável. Quando a operação é inevitável, a diminuição da agressão aos tecidos, com menor comprometimento funcional, sangramento e dor, deve ser buscada por todos; entretanto, o equilíbrio entre os riscos e benefícios é fundamental. A segurança do paciente e a precisão do procedimento não podem ser comprometidas por razões estéticas ou "mercadológicas". Sem dúvi$\mathrm{da}$, o conceito de procedimento "minimamente agressivo" é mais importante do que o de "minimamente invasivo". É verdade que as técnicas minimamente invasivas para a artroplastia total do joelho são uma realidade mas devem ser utilizadas com critérios bem definidos e por cirurgiões experientes.

\section{ARNALDO José HERNANDEZ}

Professor do Instituto de Ortopedia e Traumatologia do Hospital das Clínicas da Faculdade de Medicina da Universidade de São Paulo 


\section{REFERÊNCIAS}

1. Insall J, Ranawat C, Scott WN, Walker P. Total condylar knee replacement: preliminary report. Clin Orthop Relat Res. 1976;(120):14954.

2. Insall J, Tria AJ, Scott WN. The total condylar knee prosthesis: the first 5 years. Clin Orthop Relat Res. 1979;(145):68-77.

3. Scott RD, Volatile TB. Twelve years' experience with posterior cruciateretaining total knee arthroplasty. Clin Orthop Relat Res. 1986;(205):1007.

4. Ranawat CS, Flynn WF Jr, Saddler S, Hansraj KK, Maynard MJ. Longterm results of the total condylar knee arthroplasty. A 15-year survivorship study. Clin Orthop Relat Res. 1993;(286):94-102.

5. Malkani AL, Rand JA, Bryan RS, Wallrichs SL. Total knee arthroplasty with the kinematic condylar prosthesis. A ten-year follow-up study. J Bone Joint Surg Am. 1995;77(3):423-31.

6. Laskin RS. Reduced-incision total knee replacement through a minimidvastus technique. J Knee Surg. 2006;19(1):52-7.

7. Laskin RS. New techniques and concepts in total knee replacement. Clin Orthop Relat Res. 2003;(416):151-3.

8. Noble PC, Gordon MJ, Weiss JM, Reddix RN, Conditt MA, Mathis KB. Does total knee replacement restore normal knee function? Clin Orthop Relat Res. 2005;(431):157-65.

9. Repicci JA, Eberle RW. Minimally invasive surgical technique for unicondylar knee arthroplasty. J South Orthop Assoc. 1999;8(1):20-7; discussion 27.

10. Price AJ, Webb J, Topf H, Dodd CA, Goodfellow JW, Murray DW; Oxford Hip and Knee Group. Rapid recovery after oxford unicompartmental arthroplasty through a short incision. J Arthroplasty. 2001;16(8):970-6.

11. Pagnano MW, Meneghini RM. Minimally invasive total knee arthroplasty with an optimized subvastus approach. J Arthroplasty. 2006;21(4 suppl 1):22-6.

12. Bonutti PM, Mont MA, McMahon M, Ragland PS, Kester M. Minimally invasive total knee arthroplasty. J Bone J Surg Am. 2004;86-A Suppl 2: 26-32.

13. Scuderi GR, Tenholder M, Capeci C. Surgical approaches in miniincision total knee arthroplasty. Clin Orthop Relat Res. 2004;(428):61-7.

14. Tenholder M, Clarke HD, Scuderi GR. Minimal-incision total knee arthroplasty: the early clinical experience. Clin Orthop Relat Res. 2005; 440:67-76.

15. Cook JL, Cushner FD, Scuderi GR. Minimal-incision total knee arthroplasty. J Knee Surg. 2006;19(1):46-51.

16. Engh GA, Parks NL. Surgical technique of the midvastus arthrotomy. Clin Orthop Relat Res. 1998;(351):270-4.
17. Engh GA, Holt BT, Parks NL. A midvastus muscle-splitting approach for total knee arthroplasty. J Arthroplasty. 1997;12(3):322-31.

18. Haas SB, Manitta MA, Burdick P. Minimally invasive total knee arthroplasty: the mini midvastus approach. Clin Orthop Relat Res. 2006; 452:112-6.

19. Tria AJ Jr. Minimally invasive total knee arthroplasty: the importance of instrumentation. Orthop Clin North Am. 2004;35(2):227-34.

20. Berger RA, Deirmengian CA, Della Valle CJ, Paprosky WG, Jacobs JJ, Rosenberg AG. A technique for minimally invasive, quadriceps-sparing total knee arthroplasty. J Knee Surg. 2006;19(1):63-70.

21. Hoffman AA, Plaster RL, Murdock LE. Subvastus (Southern) approach for primary total knee arthroplasty. Clin Orthop Relat Res. 1991;(269): 70-7.

22. Roysam GS, Oakley MJ. Subvastus approach for total knee arthroplasty: a prospective, randomized, and observer-blinded trial. J Arthroplasty. 2001; 16(4):454-7.

23. Holt G, Wheelan K, Gregori A. The ethical implications of recent innovations in knee arthroplasty. J Bone Joint Surg Am. 2006;88(1):2269.

24. Laskin RS. Minimally invasive total knee arthroplasty: the results justify its use. Clin Orthop Relat Res. 2005;440:54-9.

25. Kelly MJ, Rumi MN, Kothari M, Parentis MA, Bailey KJ, Parrish WM, Pellegrini VD Jr. Comparison of the vastus-splitting and median parapatellar approaches for primary total knee arthroplasty: a prospective, randomized, study. J Bone Joint Surg Am. 2006;88(4):715-20.

26. Haas SB, Cook S, Beksac B. Minimally invasive total knee replacement through a mini midvastus approach: a comparative study. Clin Orthop Relat Res. 2004;(428):68-73.

27. Laskin RS, Becksac B, Phongjunakorn A, Pittors K, Davis J, Shim JC, et al. Minimally invasive total knee replacement through a mini-midvastus incision: an outcome study. Clin Orthop Relat Res. 2004;(428):74-81.

28. Tria AJ Jr, Coon TM. Minimal incision total knee arthroplasty: early experience. Clin Orthop Relat Res. 2003;(416):185-90.

29. Alan RK, Tria AJ. Quadriceps-sparing total knee arthroplasty using the posterior stabilized TKA design. J Knee Surg. 2006;19(1):71-6.

30. Pagnano MW, Meneghini RM, Trousdale RT. Anatomy of the extensor mechanism in reference to quadriceps-sparing TKA. Clin Orthop Relat Res. 2006;452:102-5.

31. Aglietti P, Baldini A, Sensi L. Quadriceps-sparing versus mini-subvastus approach in total knee arthroplasty. Clin Orthop Relat Res. 2006;452: 106-11

32. Lonner JH. Minimally invasive approaches to total knee arthroplasty: results. Am J Orthop. 2006;35(7 Suppl):27-9. Review. 